\title{
ÚLCERAS EM MEGACÓLONS CHAGÁSICOS OPERADOS NA URGÊNCIA E ELETIVAMENTE
}

\author{
Augusto DIOGO-FILHO ${ }^{1}$, Ademir ROCHA ${ }^{2}$, Daniel Oliveira De CONTI ${ }^{3}$ e \\ Karina Vasconcelos FERREIRA ${ }^{3}$
}

\begin{abstract}
RESUMO - Racional - O megacólon é uma doença freqüente no nosso meio e abordado na urgência pelas suas complicações como fecalomas, volvos e perfurações. As úlceras de estases nos megacólons contribuem como prováveis sítios de perfurações. Objetivo - Comparar as freqüências de úlceras de decúbito em megacólons chagásicos operados na urgência, por volvo e fecaloma, e eletivamente, objetivando melhor conduta cirúrgica na urgência. Material e métodos - Analisaram-se os laudos de 356 exames anatomopatológicos de ressecções colônicas de pacientes operados por megacólon chagásico na urgência (102 casos; $29 \%$ ) e eletivamente ( 254 casos; $71 \%$ ), no período de 1980 a 2000. As indicações cirúrgicas de urgência foram atribuídas a volvo ( 71 casos; $69,6 \%$ ), fecaloma ( 25 casos; $24,5 \%$ ), abdome agudo perfurativo após sondagem retal ou sigmoidoscopia (6 casos; 5,9\%). Compararam-se as freqüências de úlceras nos dois grupos de peças cirúrgicas, com a utilização do teste do qui-quadrado. Resultados - Nos laudos das peças cirúrgicas obtidas nas cirurgias de urgência, constatou-se o registro de úlceras em 26 casos (25,5\%); nas peças de ressecções eletivas verificaram-se úlceras em 21 casos $(8,25 \%)$. A diferença observada foi estatisticamente significante. A comparação dos grupos de volvo, fecaloma e volvo com fecaloma, em separado com o grupo das cirurgias eletivas, evidenciou diferenças significantes em relação ao volvo e ao fecaloma. Conclusão - A freqüência muito maior de úlceras nos megas operados em caráter de urgência enfatiza a necessidade da ressecção imediata do cólon sigmóide, ao invés da conduta conservadora de simples colostomia descompressiva, mesmo naquelas laparotomias exploradoras em que o exame macroscópico do sigmóide não mostre sinais de necrose. Desta forma, deve-se prevenir a ocorrência de perfuração do megacólon no pós-operatório mediato, com conseqüências usualmente graves.
\end{abstract}

DESCRITORES - Megacolo. Doença de Chagas. Úlcera varicosa. Volvo intestinal. Impacção fecal.

\section{INTRODUÇÃO}

O megacólon chagásico é abordado, cirurgicamente, tanto de forma eletiva, quanto como urgência. A abordagem cirúrgica justifica-se pelos sintomas rebeldes de constipação, assim como pelas complicações comuns e graves associadas a essa doença. O tratamento é paliativo, reduzindo a formação do fecaloma e do volvo ${ }^{(14)}$.

Dentre as abordagens eletivas, a literatura refere a retocolectomia abdominal, com abaixamento retrorretal, e anastomose colorretal retardada (cirurgia de DuhamelHaddad) como uma das técnicas mais utilizadas ${ }^{(2,7,8,15)}$, a retossigmoidectomia abdominal com anastomose mecânica colorretal término-lateral tem sido também empregada ${ }^{(5)}$, assim como a cirurgia de Duhamel realizada por via laparoscópica ${ }^{(12)}$. Nas abordagens de urgência, indicadas em casos de volvo, fecaloma não-esvaziado por lavagem e perfuração intestinal, usualmente se recomenda colostomia descompressiva em alça, ou sigmoidectomia com colostomia à Hartmann ou, ainda, sigmoidectomia com colostomia terminal em duas bocas ${ }^{(3,6,10)}$.

Em casos necropsiados de megacólon chagásico, úlceras (ditas de decúbito ou de estase) representam a complicação mais freqüentemente observada $(74,3 \%$ dos casos de megacólon complicado, na casuística de ROCHA et al. $\left.{ }^{(13)}\right)$. Entre seus possíveis fatores patogenéticos, incluise a isquemia devida à compressão da parede colônica por fecaloma ${ }^{(10)}$. As úlceras podem aprofundar-se a ponto de causar perfuração e peritonite, ou infectar-se por bactérias (fato também capaz de levar à peritonite). Assim, úlceras, com relativa freqüência, ocorrem em pacientes operados em caráter de urgência; há, todavia, muitos casos em que são observadas no exame anatomopatológico de megacólons extirpados eletivamente.

Trabalho realizado no Departamento de Cirurgia da Faculdade de Medicina e no Laboratório de Anatomia Patológica do Hospital das Clínicas da Universidade Federal de Uberlândia, MG.

${ }^{1}$ Departamentos de Cirurgia e ${ }^{2}$ Clínica Médica da Universidade Federal de Uberlândia; ${ }^{3}$ Hospital de Clínicas de Uberlândia, MG.

Endereço para correspondência: Dr. Augusto Diogo Filho - Av. Levino de Sousa, 1775 - Umuarama - 38405-322 - Uberlândia, MG. E-mail: diogofilho@netsite.com.br 
No presente trabalho, decidiu-se analisar, retrospectivamente, a freqüência de úlceras de decúbito em peças cirúrgicas de colectomias por megacólon chagásico efetuadas em caráter de urgência por volvo ou fecaloma ou eletivamente, em hospital de zona endêmica da tripanossomíase num período de 20 anos (1980 a 2000), buscando subsídios para aprimorar o tratamento cirúrgico, especialmente nas urgências. Considerando que a maioria das urgências cirúrgicas ligadas ao megacólon se deve a volvo e fecaloma, também se resolveu avaliar, em separado, as freqüências de úlceras por tais complicações, comparando-as entre si e à observada no grupo das cirurgias eletivas.

\section{MATERIAL E MÉTODO}

Analisaram-se os dados de 356 exames anatomopatológicos de megacólon chagásico realizados no período de 1980 a 2000 no Laboratório de Anatomia Patológica do Hospital de Clínicas da Universidade Federal de Uberlândia, MG.

Dos 356 casos avaliados, $196(55 \%)$ eram pacientes do sexo masculino. A idade variou de 23 a 93 anos, com média de 54 anos.

Duzentos e cinqüenta e quatro procedimentos $(71 \%)$ foram eletivos; em 102 pacientes (29\%), a cirurgia foi de urgência.

Das 102 ressecções colônicas encaminhadas à análise anatomopatológica com relato de terem sido operados na urgência, $71(69,6 \%)$ foram indicadas após volvo, 25 (24,5\%) por causa de fecaloma que não se conseguiu esvaziar através de enteroclismas por sonda retal. Em seis casos $(5,8 \%)$ a indicação cirúrgica de urgência foi por falta de êxito na abordagem do fecaloma ou volvo pela retossigmoidoscopia. Os fecalomas foram diagnosticados através de imagem radiológica ou exame físico e confirmados no ato operatório.

A abordagem dos pacientes com megacólons complicados por fecaloma ou volvo, segue protocolo bem definido no serviço de atendimento de urgência como toque retal na tentativa de fragmentação da extremidade distal do fecaloma, posicionamento de sonda retal com auxílio de retossigmoidoscopia rígida, com o objetivo de desfazer o volvo, análise do aspecto da mucosa e posicionamento de sonda retal. Nos casos em que à visibilidade endoscópica verificam-se sinais de sofrimento da mucosa colônica, o paciente é encaminhado à laparotomia de urgência.

Para a análise estatística dos resultados, utilizou-se o teste do qui-quadrado. Fixou-se em $5 \%$ ou $P<0,05$ o nível para a rejeição da hipótese de nulidade.

\section{RESULTADOS}

A relação dos procedimentos cirúrgicos realizados na urgência (102 pacientes) e eletivamente ( 254 pacientes) podem ser analisados na Tabela 1.

TABELA 1 - Distribuição dos 356 procedimentos cirúrgicos realizados de acordo com o tipo de abordagem: urgência ou eletiva

\begin{tabular}{lccc}
\hline Procedimento cirúrgico & Cirurgia de urgência & Cirurgia eletiva & Total \\
\hline Retossigmoidectomia & 26 & 202 & 228 \\
Sigmoidectomia + colostomia & 76 & 10 & 86 \\
Duhamel-Haddad & 0 & 39 & 39 \\
Colectomia subtotal & 0 & 3 & 3 \\
Total & 102 & 254 & 356 \\
\hline
\end{tabular}

A distribuição de casos com úlceras nas peças colônicas ressecadas nos grupos de pacientes submetidos a procedimento cirúrgico em caráter de urgência ou eletivamente está expressa na Tabela 2. A diferença observada entre os dois grupos foi estatisticamente significante $(P=0,0000309)$.

TABELA 2 - Distribuição dos megacólons chagásicos com úlceras em 356 procedimentos realizados de acordo com o tipo de abordagem cirúrgica (urgência ou eletiva), no Hospital de Clínicas da Faculdade de Medicina da Universidade Federal de Uberlândia, MG, no período de 1980 a 2000

\begin{tabular}{lcc}
\hline $\begin{array}{l}\text { Abordagem } \\
\text { cirúrgica }\end{array}$ & $\begin{array}{c}\text { Número de casos } \\
\mathbf{n}\end{array}$ & $\begin{array}{c}\text { Casos com úlceras } \\
\mathbf{n}(\%)\end{array}$ \\
\hline Urgência & 102 & $26(25,5 \%)$ \\
Eletiva & 254 & $21(8,25 \%)$ \\
Total & 356 & $47(13,2 \%)$ \\
\hline
\end{tabular}

$P=0,0000309$

Dos 47 megacólons chagásicos que apresentavam úlceras, 32 (68\%) pertenciam a pacientes do sexo masculino e 15 (32\%) ao do sexo feminino. A faixa etária destes pacientes variou entre 23 a 84 anos, com média de 55 anos. Dos megacólons em que se constatou úlceras, seis peças cirúrgicas (13\%) apresentavam perfuração.

A Tabela 3 mostra a ocorrência de úlceras nos megacólons operados na urgência devido a volvo e/ou fecaloma; a análise estatística entre os três grupos não mostrou diferenças estatisticamente significantes $(P>0,05)$. A comparação de cada um deles (volvo, fecaloma, volvo + fecaloma) em separado com o grupo das cirurgias eletivas, evidenciou diferenças significantes em relação ao volvo e ao fecaloma. As Figuras 1, 2 e 3 mostram aspectos macroscópicos de megassigmóide visto pela serosa, visto pela mucosa com presenças de úlceras, e o aspecto microscópico dessa úlcera, respectivamente.

TABELA 3 - Ocorrência de úlceras em megacólons chagásicos operados em caráter de urgência devido a volvo ou fecaloma, no Hospital de Clínicas da Faculdade de Medicina da Universidade Federal de Uberlândia, MG, no período de 1980 a 2000

\begin{tabular}{lcc}
\hline \multirow{2}{*}{ Complicações } & Número de casos & Casos com úlceras \\
& $\mathbf{n}$ & $\mathbf{n}(\%)$ \\
\hline Volvo & 58 & $12(20,7 \%)$ \\
Fecaloma & 25 & $7(28 \%)$ \\
Volvo com fecaloma & 13 & $2(15,4 \%)$ \\
Total & 96 & $21(22,3 \%)$ \\
\hline
\end{tabular}

$P>0,05$

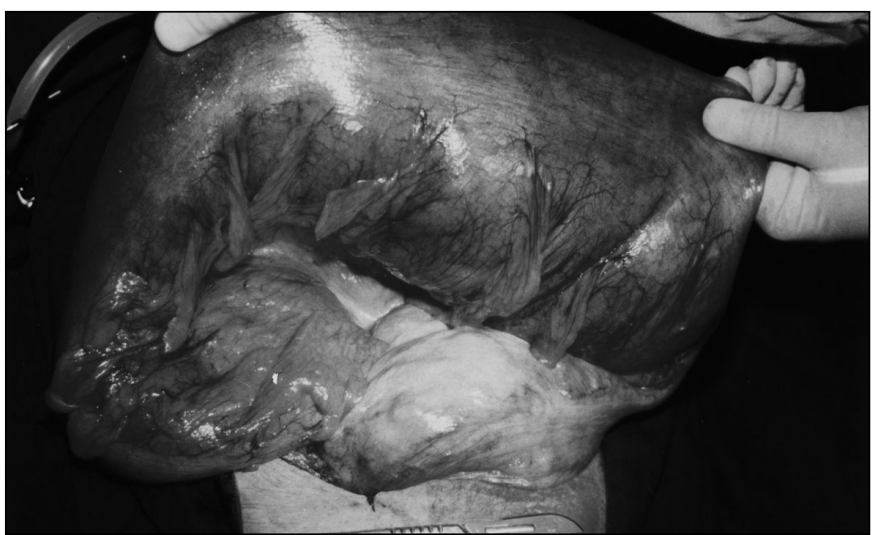

FIGURA 1 - Aspecto macroscópico de megassigmóide, em caso de volvo desfeito com aspecto normal da serosa 


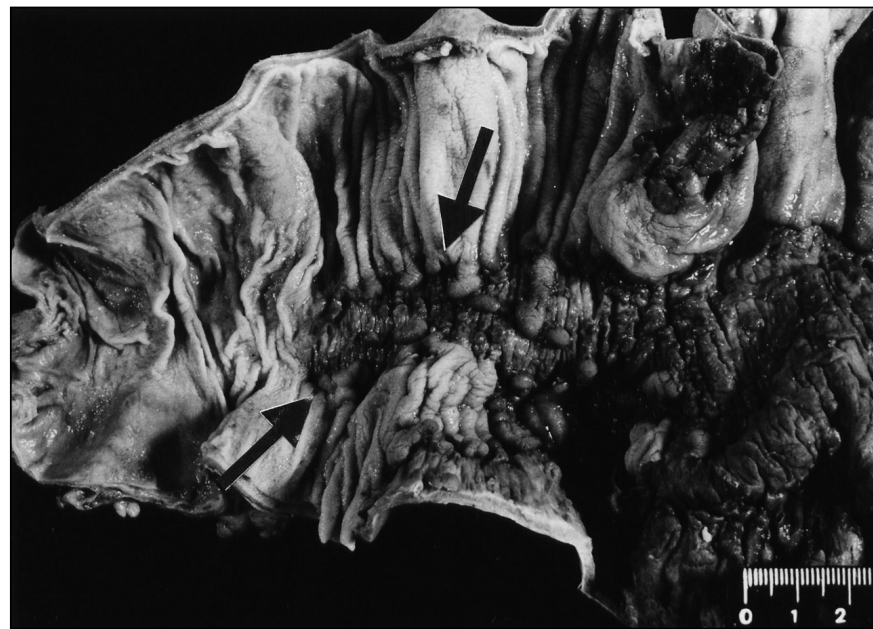

FIGURA 2 - Aspecto macroscópico de megassigmóide visto pela mucosa Presença de múltiplas úlceras (setas) de decúbito em mucosa e submucosa, que poderiam evoluir para perfuração

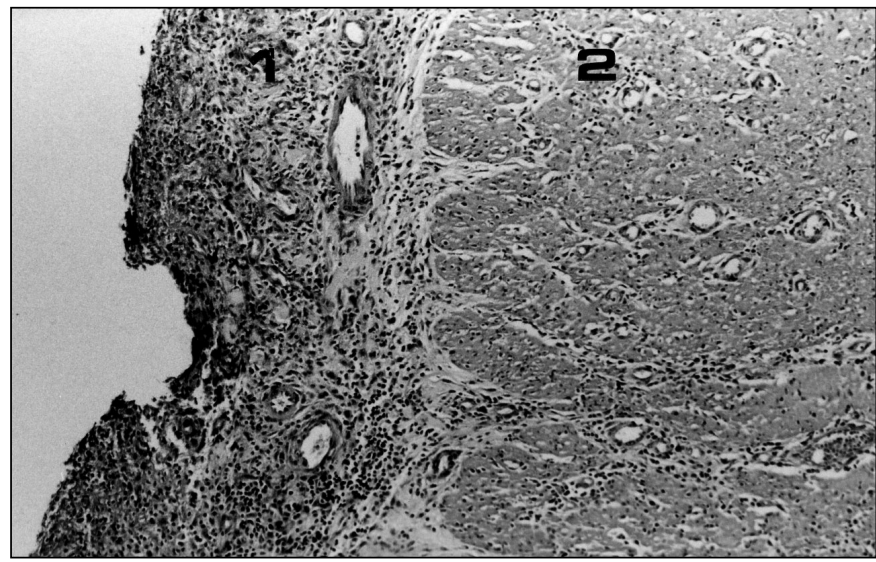

FIGURA 3 - Fotomicrografia de parede do cólon sigmóide em caso de volvo desfeito. Observar úlcera de decúbito com fundo localizado na submucosa. A mucosa foi destruída e não é observada na figura. 1- submucosa inflamada, com úlcera; 2- muscular inflamada. H-E; aumento original de 100x

\section{DISCUSSÃO}

A evolução pós-operatória dos procedimentos cirúrgicos relacionados neste estudo (Tabela 1), não é objetivo do presente trabalho e sim uma verificação macro e microscópica das peças ressecadas, já que, o objetivo principal era anotar a presença de úlceras e chamar à atenção sobre a possibilidade de provável evolução para perfuração, o que poderia ser catastrófico em pós-operatório de cirurgias de urgência nas complicações dos megacólons, quando o cirurgião não optar pela ressecção do megacólon.
A presença de úlceras ou simples exulcerações na mucosa colônica podem ser resultantes da ação traumática de fecaloma preexistente, conhecidas como úlceras estercoráceas, úlceras de contato ou de decúbito, podendo evoluir com perfuração colônica. A literatura revela incidência de $2,8 \%$ de úlceras em pacientes com de megacólon chagásico ${ }^{(9)}$.

A complicação mais freqüente do megacólon chagásico é o fecaloma. Na maioria das vezes, apresenta localização baixa, permitindo sua remoção digital associado ao uso de enteroclismas. Nos casos sem êxito com o tratamento conservador, pode ser necessário a abordagem cirúrgica do megacólon. $\mathrm{O}$ volvo de sigmóide descrito como a segunda mais freqüente complicação do megacólon, em sua maioria não apresenta áreas de necrose, permitindo a abordagem através da distorção endoscópica executada com o auxílio do retossigmoidoscópio. Na presença de necrose ou insucesso do tratamento endoscópico, o tratamento cirúrgico deverá ser realizado com caráter de urgência ${ }^{(3,14)}$.

A predominância de úlceras de decúbito nos megacólons operados em caráter de urgência, constatada no presente trabalho, verificou-se tanto em relação aos casos de fecaloma, quanto aos de volvo, comparados aos "megas" operados eletivamente.

Em relação ao fecaloma, a freqüência maior de úlceras parece ligar-se, essencialmente, à pressão exercida pela massa fecal retida contra a parede intestinal dilatada, causando isquemia e necrose; outros fatores podem contribuir para a gênese, o alargamento e o aprofundamento das lesões tais como vasculites e tromboses locais, insuficiência cardíaca, aterosclerose mesentérica concomitante e infecção bacteriana ${ }^{(11)}$.

Nos casos de volvo, a prevalência maior de úlceras pode relacionar-se a episódios prévios de torção e/ou fecaloma que teriam sido tratados clinicamente ou, nos casos de torção, corrigidos até de forma espontânea, depois de gerar isquemia, hipóxia, necrose e ulceração.

O tratamento do volvo do sigmóide através da descompressão endoscópica mostra-se bastante efetivo ${ }^{(1,6)}$. WUEPPER et al. ${ }^{(16)}$ obtiveram índice de resolução endoscópica satisfatória de $81 \%$ em 49 pacientes. No entanto, a percentagem de recidiva é alta, sendo indicada a abordagem cirúrgica do paciente na mesma internação, evitando-se assim, a vigência de novo quadro abdominal agudo $^{(4)}$.

\section{CONCLUSÕES}

Considerando a freqüência elevada de úlceras em megas com fecaloma e/ou volvo da presente casuística, parece recomendável usar, nas urgências, a ressecção obrigatória do sigmóide, independente do aspecto macroscópico perioperatório encontrado. A sigmoidectomia, já na urgência, previne a ocorrência de perfurações intestinais por aprofundamento de úlceras ou por repetição do volvo. 
Diogo-Filho A, Rocha A, De Conti DO, Ferreira KV. Ulcerations in Chagas' megacolon operated at urgency and electively. Arq Gastroenterol. 2006;43(4):280-3.

ABSTRACT - Background - The megacolon is a frequent disease in our emergencie hospital, and approached in the urgency by your complications as fecal impaction, volvulus and perforations. The ulcerations in the megacolons contribute as probable sites of perforations. Aim - To compare the frequencies of stercoral ulceration in Chagas' megacolon operated at urgency, by volvulus or fecal impaction, and electively, aiming at a better surgical conduct in the urgency surgery. Methods - It was analyzed 356 anatomy-pathological exams from colon resection of operated patients due to Chagas' megacolon at urgency (102 cases; 29\%) and electively ( 254 cases; $71 \%$ ), from 1980 to 2000. The surgical urgency indications were attributed to volvulus ( 71 cases; $69,6 \%$ ), fecal impaction ( 25 cases; $24,5 \%$ ), perforated acute abdomen after rectal catheter or sigmoidoscopy ( 6 cases; $5,9 \%$ ). The ulceration frequency was compared in both groups of resections, using chi-square. Results - The pathological anatomy - of surgery resection obtained at urgency surgeries, showed 26 cases of ulceration $(25,5 \%)$ and in electively resections were verified 21 cases of ulceration $(8,25 \%)$. The difference observed was statistically significant. The comparison among the groups of volvulus; fecal impaction and volvulus with fecal impaction, separately with electively surgery group evidenced significant differences in relation to volvulus and fecal impaction. Conclusions - The higher frequency of ulcerations in the megacolon operated at urgency character emphasizes the needs of immediate resection of sigmoid colon, instead of conservative conduct of simple decompression colostomy, even in exploration laparotomy which the macroscopic examination of sigmoid does not show necrotic signs. This way, should prevent the occurrence of perforation in megacolon at mediate postoperative, with serious results.

HEADINGS - Megacolon. Chagas disease. Varicose ulcer. Intestinal volvulus. Fecal impaction.

\section{REFERÊNCIAS BIBLIOGRÁFICAS}

1. Brunsgaard C. Volvulus of the sigmoid colon and its treatment. Surgery 1947;22:466.

2. Cutait D, Silva JH, Cutait R. Cirurgia do intestino grosso. In: Goffi FS, editor. Goffi. Técnica cirúrgica. Bases anatômicas, fisiopatológicas e técnicas da cirurgia. São Paulo: Atheneu; 1997. p.626-39.

3. Ferreira-Santos R, Carril CF, Santos Jr JCM, Guimarães AS, Aprilli F, Gomes DR. Complicações do megacolo adquirido. Rev Assoc Med Bras. 1977;23:427-8

4. Habr-Gama A, Simonsen O, Haddad J, Raia A, Corrêa-Neto A. Volvo do sigmóide - considerações gerais e resultados do tratamento conservador pelo esvaziamento endoscópico. Rev Assoc Med Bras. 1968;14:287-90.

5. Habr-Gama A, Kiss DR, Bocchini SF, Teixeira MG, Pinotti HW. Megacólon chagásico. Tratamento pela retossigmoidectomia abdominal com anastomose mecânica colorretal término-lateral. Resultados preliminares. Rev Hosp Clin Fac Med S Paulo. 1994;49:199-203.

6. Habr-Gama A, Haddad J, Montenegro ES, Simonsen O, Raia A. Volvo de sigmóide, etiologia, fisiopatologia e tratamento. Rev Hosp Clin Fac Med S Paulo. 1973;28:112-20.

7. Haddad J, Raia A, Corrêa-Neto A. Abaixamento retro-retal do cólon com colostomia perineal no tratamento do megacólon adquirido. Rev Assoc Med Bras. 1965;11:83-8.

8. Medeiros RR, Reis-Neto JA, Leonardi LS, Pires AM, Accorroni ME. Estudo comparativo entre as técnicas de Duhamel e Duhamel-Haddad na cirurgia do megacólon chagásico. Rev Paul Med. 1980;96:61-5
9. Moreira H, Rezende JM, Sebba F, Azevedo IF, Leite ACA, Soares EP. Megacolo chagásico. Rev Bras Coloproctol. 1983;3:152-62.

10. Raia AA, Habr-Gama A. Megacolo adquirido. In: Raia AA, Zerbini EJ, editores. Clínica cirúrgica Alípio Corrêa Netto. São Paulo: Sarvier; 1994. p.491-508.

11. Raia A. Anatomia patológica. In: Raia A. Manifestações digestivas da moléstia de Chagas. São Paulo: Sarvier; 1983. p.61-79.

12. Reis-Neto JA, Pedroso MA, Lupinacci RA, Reis Jr JA, Ciquini SA, Lupinacci RM, Beani Jr A, Farah JFM, Kagohara OH, Simões-Neto J, Romanholi-Neto J. Megacolo adquirido - perspectivas fisiopatológicas para o tratamento laparoscópico. Rev Bras Coloproctol. 2004;24:49-62.

13. Rocha A, Henrique D, Borges EG, Oliveira VL, Soares VMG, Moraes AT, Teixeira VPA, Almeida HO. Complicações do megacólon e megaesôfago chagásicos observadas em necropsia. Rev Goiana Med 1981;27:53-62.

14. Santos Jr JCM. Megacólon - Parte II: Doença de Chagas. Rev Bras Coloproctol. 2002;4:266-77.

15. Sousa AG, Esper FE. Tratamento cirúrgico do megacolo adquirido. Rev Col Bras Cir. 1985;12:13-7.

16. Wuepper KD, Oterman MG, Stahlgren HL. An appraisal of the operative and non operative treatment of sigmoid volvulus. Surg Gynecol Obstet. 1966;122:84.

Recebido em 6/9/2005. Aprovado em 13/7/2006. 